\title{
Cavitation instability in bulk metallic glasses
}

\author{
L.H. Dai ${ }^{1, a}$, X. Huang ${ }^{2}$, and Z. Ling ${ }^{1}$ \\ ${ }^{1}$ State Key Laboratory of Nonlinear Mechanics, Institute of Mechanics, Chinese Academy of Sciences, Beijing 100190 , \\ China \\ ${ }^{2}$ Institute of Systems Engineering, China Academy of Engineering Physics, Mianyang, Sichuan 621999, China
}

\begin{abstract}
Recent experiments have shown that fracture surfaces of bulk metallic glasses (BMGs) usually exhibit an intriguing nanoscale corrugation like fractographic feature mediated by nanoscale void formation. We attribute the onset of this nanoscale corrugation to TTZs (tension transformation zones) mediated cavitation. In our recent study, the spall experiments of Zr-based BMG using a single-stage light gas gun were performed. To uncover the mechanisms of the spallation damage nucleation and evolution, the samples were designed to be subjected to dynamic tensile loadings of identical amplitude but with different durations by making use of the multi-stress pulse and the double-flyer techniques. It is clearly revealed that the macroscopic spall fracture in BMGs originates from the nucleation, growth and coalescence of micro-voids. Then, a microvoid nucleation model of BMGs based on free volume theory is proposed, which indicates that the nucleation of microvoids at the early stage of spallation in BMGs is resulted from diffusion and coalescence of free volume. Furthermore, a theoretical model of void growth in BMGs undergoing remote dynamic hydrostatic tension is developed. The critical condition of cavitation instability is obtained. It is found that dynamic void growth in BMGs can be well controlled by a dimensionless inertial number characterizing the competition between intrinsic and extrinsic time scales. To unveil the atomic-level mechanism of cavitation, a systematic molecular dynamics (MD) simulation of spallation behaviour of a binary metallic glass with different impact velocities was performed. It is found that micro-void nucleation is determined TTZs while the growth is controlled by shear transformation zones (STZs) at atomic scale.
\end{abstract}

\section{Introduction}

Bulk metallic glasses (BMGs) are a relatively young class of alloy materials, which have amorphous atomic structure induced by fast cooling from the liquid to solid states. For the excellent physical and mechanical properties such as high strength and hardness, BMGs have potential of wide applications in the engineering areas, and has raised much attention in recent years. Especially, their fracture mechanism is widely investigated. As the atomic structures are disordered, plastic deformation of this kind of materials is prone to be localized into thin shear bands. Hence the fracture process of BMGs is usually undergoing highly localized deformation via formation and rapid propagation of shear bands [1].

However, recent studies have shown that metallic glasses can also fail by cavitation instability, which is the principle ductile fracture mechanism in conventional polycrystalline materials. Bouchaud et al. [2] conducted a quasistatic tension experiment on a $\mathrm{Zr}$-based metallic glass (Vit. 1) and typical dimple patterns were observed on the fracture surfaces of tested samples. Through the study of the mismatch between two opposite fracture surfaces, it is shown that the fracture is controlled by growth and coalescence of damage cavities. To reveal the fracture mechanism of bulk metallic glasses, Jiang et al. [3] performed a systematic study on fracture patterns of Vit. 1 via compression, tensile and plate impact experiments. Dimple and honeycomb structures were observed on

\footnotetext{
${ }^{\text {a }}$ Corresponding author: 1 hdai@lnm.imech.ac.cn
}

dynamic mode I fracture surfaces, which indicated that the fracture is a void dominated process. Particularly, fine $100 \mathrm{~nm}$ size dimples and nanoscale periodic corrugations were found as well. Based on the fracture patterns, a new atomic motion unit called as tension transformation zone (TTZ), which may be interpreted as nucleation of nanoscale voids ahead of crack tip, was proposed. Similar phenomenon has been observed in spallation experiments studied the spallation behaviour of Zr-based metallic glass (Vit. 1) via plate-impact experiments. Equiaxed cellular patterns were found on the spall surface of recovered samples, which indicated that the spallation in the materials is induced by nucleation, growth and coalescence of micro-voids. Moreover, this micro-voids dominated fracture process has also been observed in atomic scale during molecular dynamics simulations. Arman et al. [4] investigated the dynamic response of a $\mathrm{CuZr}$ metallic glass with MD simulations and found that spallation process of this material is accompanied by nucleation and growth of micro-voids. In order to reveal the intrinsic mechanism that governs the fracture process in different metallic glasses, Murali et al. [5] performed MD simulations on two typical metallic glasses which were brittle FeP and ductile CuZr. Their work showed that even the brittle FeP glass can fracture through nucleation and coalescence of multiple voids for the high degree of atomic scale spatial fluctuations.

It is clear that void dominated fracture process is a common failure mode in metallic glasses. To understand the fracture mechanism, our recent experimental results 
are reported in this paper. Some theoretical models are proposed to reveal the void nucleation and growth mechanism. Furthermore, MD simulations of spallation behaviour of a binary metallic glass are perform, which show the cavitation mechanism at atomic level.

\section{Spallation experiments}

To obtain the physical picture of cavitation instability, spallation experiments are conducted on a single-stage light gas gun. The sample material we used was a $\mathrm{Zr}$ based amorphous alloy with the nominal composition $\mathrm{Zr}_{41.2} \mathrm{Ti}_{13.8} \mathrm{Cu}_{12.5} \mathrm{Ni}_{1} 0 \mathrm{Be}_{22.5}$ (Vit. 1). The flyer material was H62 brass, which is similar to C28000 brass (e.g., $62 \%$ $\mathrm{Cu}$ and $38 \% \mathrm{Zn}$ ).

To obtain stress pulses with the identical amplitude and different durations, a double-flyer technique is used. With this technique, the samples are impacted by two flyers, and the stress durations are controlled accurately by the gap between them. In our experiments, the impact velocity was $200 \mathrm{~m} / \mathrm{s}$, corresponding to a stress amplitude of $\sim 3.2 \mathrm{GPa}$. And the stress durations are controlled to be $125 \mathrm{~ns}, 250 \mathrm{~ns}$ and $372 \mathrm{~ns}$.

Close-up observations with a high resolution scanning electronic microscopy (HRSEM) (FEI-Sirion NC HRSEM with $1.5 \mathrm{~nm}$ resolution) were performed to recovered samples. The spallation damage evolution process was observe in the recovered BMG samples. While the stress duration increases, there are more micro-cracks observed on the cross-section. Details of the cracks shows that they are formed due to nucleation, growth and coalescence of micro-voids. Now, the question is what is mechanism of void nucleation and evolution in BMGs.

\section{Void nucleation mechanism}

To understand the void nucleation mechanism, we proposed a we propose a void nucleation model of amorphous alloys developed from traditional stress temperature-driven models widely used in polycrystalline materials [6]. We also consider free-volume theory to understand and characterize microvoid nucleation in BMGs.

In our model, the process of void nucleation can be divided into three stages: First, the frozen free volume is activated by applied tensile stress; second, free volume diffuses in the material and causes a net diffusional coalescence; and third, a void that is larger than a critical size forms and can grows under tensile loading as shown in Fig. 1. At this stage, the void is nucleated.

Based on the model, we derived the nucleation rate theoretically written as [6]:

$$
\dot{N}=\frac{A}{\sigma_{T}^{2}} \exp \left(B \sigma_{T}-\frac{C}{\sigma_{T}^{3}}\right)
$$

where $\sigma_{T}$ the applied tensile stress under uniaxial strain condition, $A$ is a material constant at a certain temperature, $B$ describes the influence of tensile loading on the activation barrier, $\mathrm{C}$ is a coefficient associated with the influence of tensile loading on the number of subcritical nuclei.

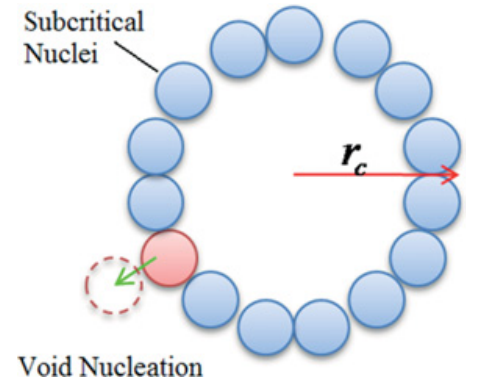

Figure 1. Schematic of void nucleation [6].

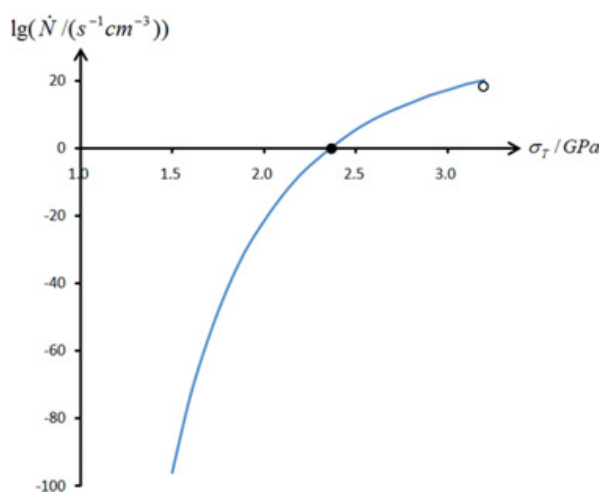

Figure 2. Schematic of void nucleation [6].

To test the reliability of our void nucleation model, we choose the BMG Vit. 1 as a model material. Using typical values of the material parameters, we obtain the curve of nucleation rate as a function of the applied tensile stress, as shown in Fig. 2.

If the value zero on the vertical scale in Fig. 2 is designated as the critical value below which void nucleation is so slow that it can be neglected, the threshold stress of void nucleation for Vit. 1 is about $2.35 \mathrm{GPa}$, a little higher than the quasistatic tensile strength of Vit.1, $1.86 \mathrm{GPa}$. As the spall strength is usually thought to be higher than the quasistatic tensile strength because of the loading rate effect, the threshold stress obtained by Eq. (3) is reasonable. We also compared the calculated result with our experimental data. From the observed cellular pattern, we estimated the nucleation rate (marked as a white point in Fig. 2) in our spallation experiments. It is obvious that the theoretical result obtained by Eq. (1) agrees quite well with the experimental data.

\section{Void growth mechanism}

Next, void growth in BMGs under remote tensile loading was investigated [7].

\subsection{Basic model}

We consider a spherical void of radius $a$ in an infinite metallic glass under the remote hydrostatic tensile pressure $p^{a p p}$, as shown in Fig. 3. The void grows as the hydrostatic tensile pressure is applied. It is assumed that the void remains spherical throughout the growth process. Once the void surface yields, the elastic-viscoplastic boundary is considered as a spherical surface propagating outwards. 


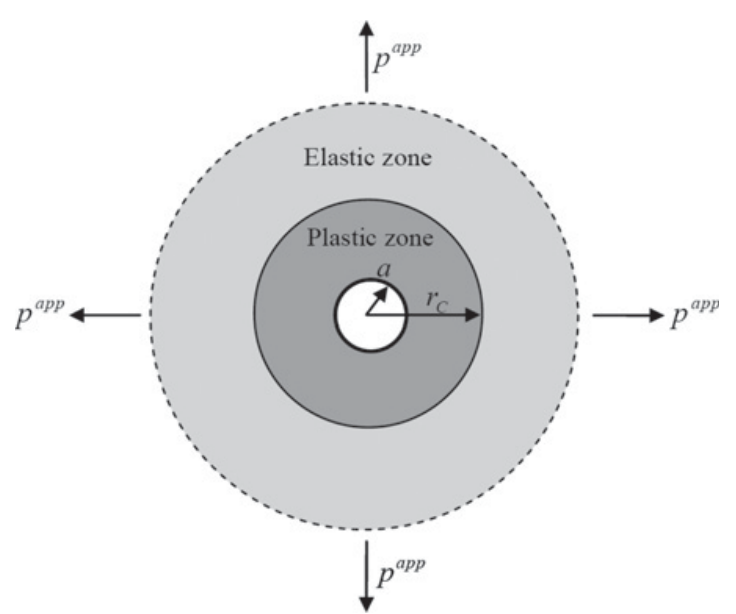

Figure 3. Schematic of void growth under tensile pressure [7].

Then, according to continuum mechanics and void growth model in conventional polycrystalline materials, we can write the governing equations [7]. Here we just give some key equations.

To describe the matrix material (BMG), an elasticviscoplastic constitutive law is adopted as:

$$
\begin{gathered}
\varepsilon_{i j}=\frac{s_{i j}}{2 \mu} \text { if } \tau+Q p \leq \hat{\tau}-\left(C_{1} T / T_{g}\right)^{1 / 2} \\
\dot{\varepsilon}_{i j}=\frac{\dot{s}_{i j}}{2 \mu}+\frac{s_{i j}}{2 \eta} \text { if } \tau+Q p \geq \hat{\tau}-\left(C_{1} T / T_{g}\right)^{1 / 2}
\end{gathered}
$$

where $\varepsilon_{i j}$ is the strain tensor, $s_{i j}$ is the stress deviator, $\mu$ is the shear modulus, and $\eta$ is the viscosity. To characterize the pressure sensitivity in plastic flow, the yield criterion of BMGs suggested by Sun et al. [8] is used. In this criterion, $\tau=\left(\sigma_{\theta}-\sigma_{r}\right) / 2$ is the maximum shear stress ( $\sigma_{\theta}$ and $\sigma_{r}$ are the principle stresses), $p=\left(\sigma_{r}+2 \sigma_{\theta}\right) / 3$ is the hydrostatic pressure, $\mathrm{Q}$ is the pressure sensitivity coefficient and the term $\hat{\tau}-\left(C_{1} T / T_{g}\right)^{1 / 2}$ represents the yield strength ( $\hat{\tau}$ is the barrier shear resistance of a STZ, $C_{1}$ is a coefficient that reflects the temperature dependence of strength, $T$ is the temperature, and $T_{g}$ is the glass transition temperature).

And the stress dependent viscosity is defined as:

$$
\eta \equiv \frac{\tau_{e}}{\dot{\gamma}^{p}}=\frac{\tau_{e}}{2 f \exp \left(-\frac{\Delta G^{m}}{k_{B} T}\right) \sinh \left(\frac{\tau^{e} \Omega}{2 k_{B} T}\right) \exp \left(-\frac{1}{\xi}\right)}
$$

where $\tau_{e}=\sqrt{J_{2}}=\sqrt{s_{i j} s_{i j} / 2}$ is the effective shear stress, $\dot{\gamma}^{p}$ is the plastic strain rate, $f$ is the frequency of atomic vibration ( $\sim$ Debye frequency), $\Delta G^{m}$ is the activation energy, $k_{B}$ is the Boltzmann constant, $\Omega$ is the atomic volume, and $\xi$ is the concentration of free volume $(\xi=$ $v_{f} / \chi v^{*}$, here $v_{f}, \chi$ and $v^{*}$ are, respectively, the free volume, a geometric factor and the effective hard-sphere size of an atom).

The free volume concentration is adopted as an order parameter, which is a function of the radius $r$ in the current configuration and the time $t$. The free volume evolution

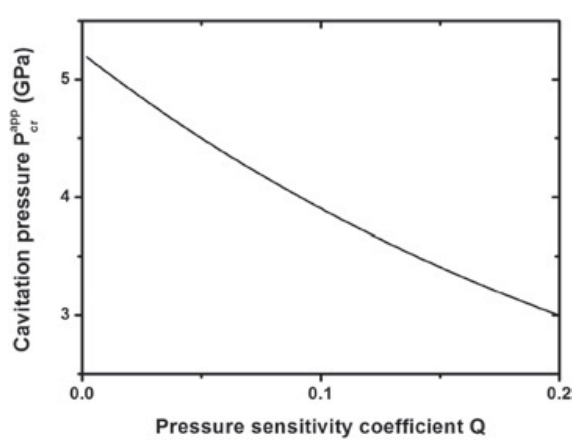

Figure 4. The cavitation pressure as a function of pressure sensitivity coefficient [7].

equation is written as:

$$
\frac{\partial \xi}{\partial t}=D \nabla^{2} \xi+G(\xi, T, \tau, p)
$$

where $D$ is the diffusion coefficient of free volume concentration and $G(\xi, T, \tau, p)$ is the net generation rate of free volume.

\subsection{Cavitation instabilities}

Cavitation instability can be regarded as the unbounded growth of a pre-existing void. Usually a critical pressure determines whether cavitation instability occurs. To get out this criterion, we consider the quasistatic case, and derived the critical pressure in BMGs as follows [7]:

$$
\begin{aligned}
p_{c r}^{a p p}= & \frac{\hat{\tau}-\left(C_{1} T / T_{g}\right)^{1 / 2}}{Q} \\
& -\frac{1}{Q \mu^{4 Q / 3}}\left(\frac{3 \hat{\tau}-3\left(C_{1} T / T_{g}\right)^{1 / 2}}{3+4 Q}\right)^{(3+4 Q) / 3} .
\end{aligned}
$$

Using the material parameters of a typical metallic glass Vit. 1, the critical pressure for cavitation instabilities versus the pressure sensitivity coefficient is shown in Fig. 4. We found that the cavitation pressure decreases as the pressure sensitivity coefficient increases.

\subsection{Dynamic growth of voids}

To reveal the dominant factor that controls the void growth, we proposed an dimensionless number by scaling the governing equations [7]:

$$
I_{\text {inertia }}=\frac{\sqrt{\rho A^{2} / p_{S}}}{t_{a}}+\frac{\sqrt{\rho A^{2} / p_{S}}}{\eta / \mu} .
$$

Here, $I_{\text {inertia }}$ is a dimensionless number similar to the Deborah number and consists of three characteristic time scales: (1) the inertial time scale $t_{\text {inertia }}=A / \sqrt{p_{S} / \rho}$, a characteristic time scale of the outward flux of matter; (2) the loading time scale $t_{a}$, which is just the rise time of the loading history; and (3) the relaxation time scale $t_{r}=$ $\eta / \mu$, a characteristic time for viscous flow. The three time scales represent inertial, loading rate and viscous effects respectively. 
To quantify the effects of dominant factors, finite difference method simulations on dynamic void growth were carried out. The loading which is applied on the outer boundary is divided to two stages: the rise stage in which the applied pressure increases linearly until the desired loading $p_{S}$ is achieved after a rise time $t_{a}$, and the steady stage in which the loading is held constant at $p_{S}$ during the hold time $t_{S}$. It is found that at the rise stage of loading history, the competition between the inertial effects and the loading rate effects controls the growth process. While the inertial effects become more dominant, the vibration of growth rate is observed. As the viscous effects begin to work, the loading rate effects are gradually replaced, and the viscous effects will induce higher growth rate and disappearance of vibrating growth. At the steady stage, the competition between the inertial effects and the viscous effects controls the growth process. While the inertial effects become dominant, the growth of void is obviously impeded.

\section{Atomic-level mechanism of cavitation}

To unveil the atomic-level mechanism of cavitation, a systematic molecular dynamics (MD) simulation of spallation behavior of a binary metallic glass was performed [9].

\subsection{MD simulation of spallation}

We choose a simple binary $\mathrm{MG} \mathrm{Zr}_{50} \mathrm{Cu}_{50}$ as the model material. To describe the atomic interactions, the FinnisSinclair type interatomic potential with parameters given by Mendelev et al. [10] is used. Calculations are carried out with the open source code LAMMPS. For simulations of spallation, we construct the traditional flyer-target configurations. The size of flyer plate is about $100 \times 20 \times$ $20 \mathrm{~nm}^{3}$, and the target has the same cross-section area $(20 \mathrm{~nm} \times 20 \mathrm{~nm})$ but its thickness is twice as that of flyer. To achieve shock loading with different amplitudes, we choose different impact velocities $V$ of $600,900,1200$, 1500 , and $1800 \mathrm{~m} / \mathrm{s}$ respectively.

Figure 5 illustrates the free surface velocity histories on the target side. Typical "pull-back" waves, which are signatures for spallation, are observed in all cases, except the case of $\mathrm{V}=600 \mathrm{~m} / \mathrm{s}$. It indicates that spallation has occurred in the cases of $\mathrm{V}=900,1200,1500$, and $1800 \mathrm{~m} / \mathrm{s}$. To reveal the spallation mechanism at atomic level, the case of $\mathrm{V}=900 \mathrm{~m} / \mathrm{s}$ is further characterized.

\subsection{Atomic-level mechanism}

Figure 6 shows the damage evolution process at $\mathrm{V}=$ $900 \mathrm{~m} / \mathrm{s}$, and the colours represent value of $D_{\min }^{2}$ calculated from the same reference $(\mathrm{t}=81 \mathrm{ps})$. It is clear that the spallation occurs via nucleation and growth of voids in the MG.

Here the critical size for void nucleation is determined to be $\sim 1 \mathrm{~nm}$ in diameter. Thus, according to the void size, Figs. $6(\mathrm{a}-\mathrm{c})$ illustrate the nucleation process, but Figs. 6(d-e) exhibit the growth process. As shown in Figs. 6(a-c), during the void nucleation process, the atoms with a larger nonaffine displacement are randomly

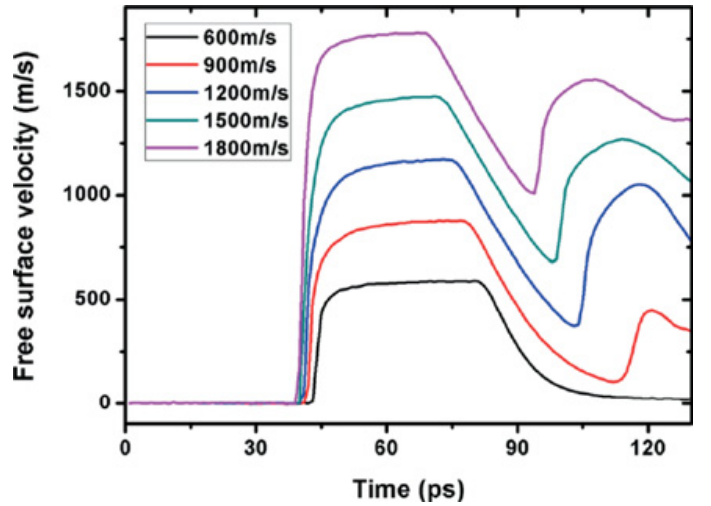

Figure 5. Damage evolution process at $\mathrm{V}=900 \mathrm{~m} / \mathrm{s}$ [9].

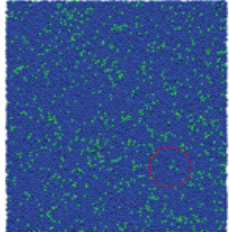

(a) $\mathrm{t}=83 \mathrm{ps}$

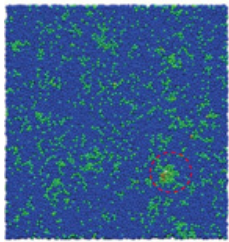

(d) $\mathbf{t}=89 \mathrm{ps}$

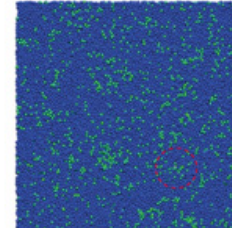

(b) $\mathbf{t}=85 \mathrm{ps}$

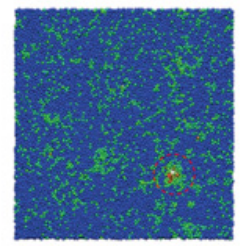

(e) $t=90 \mathrm{ps}$

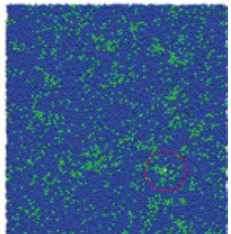

(c) $t=87 \mathrm{ps}$

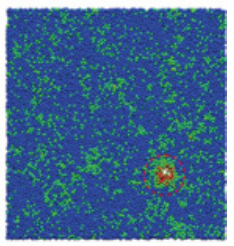

(f) $\mathrm{t}=91 \mathrm{ps}$
Figure 6. Damage evolution process at $\mathrm{V}=900 \mathrm{~m} / \mathrm{s}$ [9].

distributed in the material. With increasing the time interval (the reference configuration is the same), the number of atoms with larger nonaffine displacement increases. No apparent difference is observed between the location where void nucleation occurs and other region. It implies that the nonaffine displacement is induced by temperature (or structural relaxation) instead of stress. However, during the void growth process, nonaffine displacement of atoms in the region around the void is much larger than that away from the void. It indicates that plastic deformation of the material is mainly induced by void growth, there is nearly no contribution from void nucleation.

Further investigation reveals that the nucleation and growth of voids is closely related to the fundamental unitprocesses of collective atomic motion in MGs. Figure 7 shows some close-up views of the atomic cluster motion around the void. As shown in Fig. 7(a), during the nucleation process, transformation of the atomic structure at the centre of the void is corresponding to the picture of tension transformation zone (TTZ) proposed by Jiang et al. [3] But during the growth process as shown in Fig. 8(b), the motion of the atomic cluster at the edge of the void is close to the picture of shear transformation zone (STZ) proposed by Argon et al. [11] Thus, we argue that cavitation instability originates from TTZs and develops from STZs at atomic scale. 
(a) Nucleation-Activation of TTZ

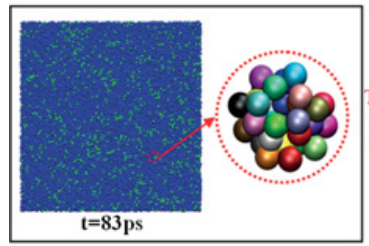

$\mathbf{t}=83 \mathrm{ps}$

(b) Growth - Activation of STZ
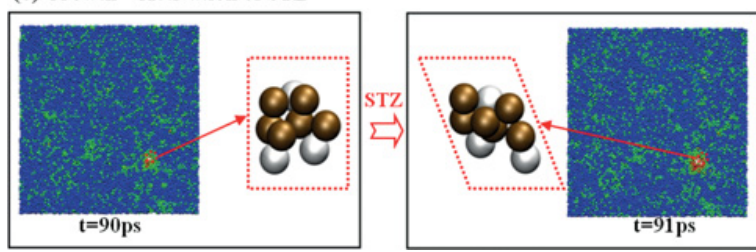

Figure 7. Motion of atomic clusters during the damage evolution process at $\mathrm{V}=900 \mathrm{~m} / \mathrm{s}$ : (a) nucleation; and (b) growth [9].

\section{Conclusion}

In this paper, we have investigated cavitation instability in MGs. Our recent experimental results shows that spallation in BMGs is due to nucleation, growth and coalescence of micro-voids. Based on free volume theory, a void nucleation model is proposed, and the nucleation can be interpreted as a diffusion and coalescence process of free volume. Theoretical and numerical study of void growth reveals that the void growth rate is controlled by inertial, loading rate and viscous effects. Via MD simulations, we found that micro-void nucleation is determined TTZs while the growth is controlled by shear transformation zones (STZs) at atomic scale.
Financial support was from the National Key Basic Research Program of China (2012CB937500), the NSFC (Grants Nos.: 11272328, 11472287, and 11402245), and the CAS/SAFEA International Partnership Program for Creative Research Teams.

\section{References}

[1] L.H. Dai, M. Yan, L.F. Liu and Y.L. Bai, Appl. Phys. Lett. 87 (14), 141916 (2005).

[2] E. Bouchaud, D. Boivin, J.L. Pouchou, D. Bonamy, B. Poon and G. Ravichandran, Europhys. Lett. 83 (6), 66006 (2008).

[3] M.Q. Jiang, Z. Ling, J.X. Meng and L.H. Dai, Philos. Mag. 88 (3), 407-426 (2008).

[4] B. Arman, S.-N. Luo, T.C. Germann and T. Çağın, Phys. Rev. B 81 (14), 144201 (2010).

[5] P. Murali, T. Guo, Y. Zhang, R. Narasimhan, Y. Li and H. Gao, Phys. Rev. Lett. 107 (21), 215501 (2011).

[6] X. Huang, Z. Ling, H.S. Zhang, J. Ma and L.H. Dai, J. Appl. Phys. 110 (10), 103519 (2011).

[7] X. Huang, Z. Ling and L.H. Dai, Int. J. Solids Struct. 50 (9), 1364-1372 (2013).

[8] L. Sun, M.Q. Jiang and L.H. Dai, Scripta Mater. 63 (9), 945-948 (2010).

[9] X. Huang, Z. Ling and L.H. Dai, J. Appl. Phys. 116, 143503 (2014).

[10] M.I. Mendelev, D.J. Sordelet and M.J. Kramer, J. Appl. Phys. 102(2), 043501 (2007).

[11] A.S. Argon, Acta Metall. 27(1), 47-58 (1979). 